\begin{abstract}
Aus der Klinik mit Schwerpunkt Hämatologie, Onkologie und Tumorimmunologie am Campus Mitte der Medizinischen Fakultät Charité - Universitätsmedizin Berlin
\end{abstract}

\title{
DISSERTATION
}

\section{Influence of CYP2D6 genotype on tamoxifen efficacy in advanced breast cancer}

\author{
zur Erlangung des akademischen Grades \\ Doctor medicinae (Dr. med.)
}

\author{
vorgelegt der Medizinischen Fakultät \\ Charité - Universitätsmedizin Berlin
}

von

Jennifer Karle

aus Ludwigsfelde

Datum der Promotion: $\quad$ 22.06.2014 


\section{Inhaltsverzeichnis}

Zusammenfassung $\quad 1$

Abstract 3

Eidesstattliche Versicherung / Anteilserklärung 4

Auszug aus der Journal Summary List $\quad 6$

$\begin{array}{lr}\text { Publikation } & 9\end{array}$

$\begin{array}{ll}\text { Lebenslauf } & 17\end{array}$

$\begin{array}{ll}\text { Publikationsliste } & 20\end{array}$

$\begin{array}{ll}\text { Danksagung } & 21\end{array}$ 


\section{Zusammenfassung}

\section{Einleitung}

Der Einfluss des CYP2D6-Genotyps auf die Wirksamkeit von Tamoxifen (Tam) wurde beim Mammakarzinom im Frühstadium mit widersprüchlichen Ergebnissen und großer Heterogenität bezüglich des Studiendesigns, der Methodik und der Komedikation untersucht. Allerdings gibt es bisher nur zwei Studien, die über den potenziellen Einfluss beim fortgeschrittenen Mammakarzinom berichten. Unsere Fragestellung war demnach, inwieweit die palliative Therapie mit Tam bei Patienten mit einem funktionellen CYP2D6-Allel wirksamer ist, als bei Individuen mit zwei funktionslosen Allelen.

\section{Patienten und Methoden}

Inkludiert wurden Patienten mit fortgeschrittenem, Hormonrezeptor-positivem (Östrogen- und / oder Progesteronrezeptoren) Mammakarzinom, die eine palliative Therapie mit Tam in Standarddosierung $(20 \mathrm{mg} / \mathrm{d})$ erhielten oder bereits erhalten hatten. Patienten mit simultaner Einnahme von CYP2D6-Inhibitoren wurden aus der Studie ausgeschlossen. Die genomische DNA wurde aus Vollblut $(\mathrm{n}=51)$ und Formalin-fixiertem, Paraffin-eingebettetem (FFPE) Gewebe $(\mathrm{n}=43)$ isoliert. In den Vollblutproben wurden die Allele $C Y P 2 D 6 * 2, * 3, * 4, * 5$, *6, $* 10, * 17, * 29, * 41$, die CYP2D6-Duplikation / Multiplikation sowie CYP2D6*4 im FFPEGewebe bestimmt. Primärer Endpunkt war das progressionsfreie Überleben (PFS), sekundäre Endpunkte waren u.a. der klinische Nutzen (CB) und das Gesamtüberleben (OS). Die Durchführung der Genotypisierung wurde verblindet und die klinischen Daten separat analysiert.

\section{Ergebnisse}

In die Studie wurden 94 Patienten mit einem medianen Alter von 59 Jahren (29 - 90 Jahre) eingeschlossen. Bei 6 Patienten gelang keine eindeutige Genotypisierung, sodass die Proben von der weiteren Analyse ausgeschlossen wurden. Die Genotypisierung ergab folgende Ergebnisse: $1,1 \%$ UMs, 84,1 \% EMs, 3,4 \% IMs und 11,4\% PMs. Patienten ohne voll funktionsfähiges Allel (IM/IM, IM/PM, PM/PM) hatten ein signifikant kürzeres PFS und OS im Vergleich zu Patienten mit mindestens einem funktionalen Allel (EM/EM, EM/IM, EM/PM) (PFS: p = 0,017; $\mathrm{HR}=2,19,95 \%$-KI, 1,15-4,18; OS: $\mathrm{p}=0,028$; HR = 2,79, $95 \%$-KI, 1,12-6,99). Die CB-Rate betrug $73 \%$ für die EM-Gruppe und 38,5\% für IM + PM-Gruppe (p = 0,019). 


\section{Schlussfolgerung}

Unsere Ergebnisse zeigen einen signifikanten Einfluss des CYP2D6-Genotyps auf die Wirksamkeit von Tam in der Behandlung des fortgeschrittenen Mammakarzinoms. Im Gegensatz zur adjuvanten Therapie ist die bisher publizierte Datenlage in der palliativen Situation kongruent. Ursachen für die widersprüchlichen Ergebnisse der adjuvanten Studien sind vermutlich auch die wesentlichen methodischen Unterschiede zwischen den Publikationen und die Auswahl des Patientenkollektivs. Unseres Erachtens sollte die Bestimmung des CYP2D6Genotyps bei fortgeschrittenem Mammakarzinom vor Einleitung einer Therapie mit Tam erwogen und die gleichzeitige Einnahme von CYP2D6-Inhibitoren vermieden werden. 


\section{Abstract}

\section{Purpose}

The influence of CYP2D6 genotype on efficacy of tamoxifen (Tam) has been extensively analyzed in early breast cancer with conflicting results and large differences concerning study design, allelic determination and co-medication. However, there is only scarce data regarding this potential influence in advanced breast cancer (ABC). We hypothesize that Tam is more effective in patients with a functional CYP2D6 allele than without any fully functional allele.

\section{Patients and Methods}

ABC patients with prior or ongoing palliative Tam treatment $(20 \mathrm{mg} / \mathrm{d})$ and verified positive hormone receptor status (ER and / or PR) were eligible. Comedication with CYP2D6 inhibitors during the time that Tam was used for metastatic disease was an exclusion criterion. Genomic DNA was extracted from blood $(\mathrm{n}=51)$ and formalin-fixed, paraffin-embedded (FFPE) tissue (n $=43)$. CYP2D6*2, *3, *4, *5, *6, *10,*17,*29,*41, CYP2D6 duplication and multiplication were determined in blood and $C Y P 2 D 6 * 4$ in FFPE-tissue. Primary endpoint was progression free survival (PFS); secondary endpoints included clinical benefit (CB), and overall survival (OS). Genotyping was performed blinded and clinical data were analyzed separately.

\section{Results}

94 patients were identified with a median age of 59 y (29 - 90 y). In 6 patients genotyping did not show conclusive results and were therefore excluded from further analysis. Genotyping results were as follows: $1.1 \% \mathrm{UMs}, 84.1 \% \mathrm{EMs}, 3.4 \% \mathrm{IMs}$ and $11.4 \%$ PMs. Patients without any fully functional allele (IM/IM, IM/PM, PM/PM) had a significant shorter PFS and OS compared to patients with at least one functional allele (EM/EM, EM/IM, EM/PM) (PFS: p = $0.017 ; \mathrm{HR}=2.19 ; 95 \%$-CI, 1.15-4.18; OS: $\mathrm{p}=0.028 ; \mathrm{HR}=2.79 ; 95 \%$-CI, 1.12-6.99). The CB rate was $73 \%$ for EM-group and $38.5 \%$ for IM + PM-group (p = 0.019).

\section{Conclusions}

Our results show a significant influence of CYP2D6 genotype on efficacy of Tam in the treatment of $\mathrm{ABC}$. In contrast to the adjuvant setting evidence in the palliative setting is congruent. The reasons for this are probably the methodological differences and the selection of the patient population of adjuvant trials. CYP2D6 testing in ABC should be considered and the concomitant use of CYP2D6 inhibitors avoided. 


\section{Eidesstattliche Versicherung / Anteilserklärung}

„Ich, Jennifer Karle, versichere an Eides statt durch meine eigenhändige Unterschrift, dass ich die vorgelegte Dissertation mit dem Thema:

-Influence of CYP2D6 genotype on tamoxifen efficacy in advanced breast cancer-

selbstständig und ohne nicht offengelegte Hilfe Dritter verfasst und keine anderen als die angegebenen Quellen und Hilfsmittel genutzt habe.

Alle Stellen, die wörtlich oder dem Sinne nach auf Publikationen oder Vorträgen anderer Autoren beruhen, sind als solche in korrekter Zitierung (siehe „Uniform Requirements for Manuscripts (URM)“ des ICMJE -www.icmje.org) kenntlich gemacht. Die Abschnitte zu Methodik (insbesondere praktische Arbeiten, Laborbestimmungen, statistische Aufarbeitung) und Resultaten (insbesondere Abbildungen, Graphiken und Tabellen) entsprechen den URM (s.o) und werden von mir verantwortet.

Mein Anteil an der ausgewählten Publikation entspricht dem, der in der untenstehenden gemeinsamen Erklärung mit dem Betreuer, angegeben ist.

Die Bedeutung dieser eidesstattlichen Versicherung und die strafrechtlichen Folgen einer unwahren eidesstattlichen Versicherung ( $\$ 156,161$ des Strafgesetzbuches) sind mir bekannt und bewusst.“

Datum

Unterschrift

\section{$\underline{\text { Anteilserklärung an der erfolgten Publikation }}$}

Jennifer Karle hatte folgenden Anteil an der folgenden Publikation:

Karle J, Bolbrinker J*, Vogl S, Kreutz R, Denkert C, Eucker J, Wischnewsky M, Possinger K, Regierer AC: Influence of CYP2D6 genotype on tamoxifen efficacy in advanced breast cancer. Breast Cancer Res Treat 2013;139:553-60.

*Juliane Bolbrinker ist ebenfalls Erstautorin dieser Publikation und hat bereits promoviert. 


\section{Beitrag im Einzelnen:}

- Beteiligung an Idee, Konzept und Design der Studie

- Durchsicht diverser medizinischer Datenbanken zur Identifizierung geeigneter Patienten

- Inklusion geeigneter Patienten

- Organisation der Patientenaufklärung

- Beteiligung an der Blutprobengewinnung

- Archivrecherche im Pathologischen Institut der Charité

- Selektion und Anforderung geeigneter Paraffinblöcke

- Organisation der pathologischen Begutachtung und Anfertigung der Gewebeproben

- DNA-Isolierung aus Vollblut und Gewebe

- Durchführung von drei unterschiedlichen Methoden zur Bestimmung des CYP2D6Genotyps (PCR, Real-time PCR, Präamplifikation)

- Analyse der Ergebnisse und Fehlersuche

- Optimierung der Versuchsbedingungen für die Genotypisierung des FFPE-Gewebes

- Beteiligung an der Interpretation der Ergebnisse

- Beteiligung an der Erhebung der Patientendaten

- Beteiligung an statistischer Auswertung

- Literaturrecherche

- Erstellung und Überarbeitung des Manuskripts

Unterschrift, Datum und Stempel des betreuenden Hochschullehrers

Unterschrift der Doktorandin 


\section{Auszug aus der Journal Summary List}

Das Journal „Breast Cancer Research and Treatment” gehört zu den international führenden onkologischen Fachzeitschriften und belegt aktuell (11.06.2013) den 37. Rang (37/196). Die einzelnen Daten sind in den folgenden Tabellen aufgelistet.

Tabelle 1: ISI Web of KnowledgeSM. Marked Journal list.

\begin{tabular}{|c|c|c|c|c|c|c|c|c|c|}
\hline $\begin{array}{c}\text { Abbreviated } \\
\text { Journal } \\
\text { Title }\end{array}$ & ISSN & $\begin{array}{l}2011 \\
\text { Total } \\
\text { Cites }\end{array}$ & $\begin{array}{c}\text { Impact } \\
\text { Factor }\end{array}$ & \begin{tabular}{|c||} 
5-Year \\
Impact \\
Factor
\end{tabular} & $\begin{array}{c}\text { Immediacy } \\
\text { Index }\end{array}$ & $\begin{array}{c}2011 \\
\text { Articles }\end{array}$ & \begin{tabular}{|c|} 
Cited \\
Half- \\
life
\end{tabular} & $\begin{array}{c}\text { Eigenfactor } \\
\text { Score }\end{array}$ & $\begin{array}{c}\text { Article } \\
\text { Influence }^{\circledR} \\
\text { Score } \\
\end{array}$ \\
\hline $\begin{array}{l}\text { BREAST } \\
\text { CANCER RES } \\
\text { TR } \\
\end{array}$ & $\begin{array}{l}0167- \\
6806\end{array}$ & $\mid 12507$ & 4.431 & 4.351 & 0.955 & 554 & 4.0 & 0.04431 & 1.392 \\
\hline
\end{tabular}




\section{ISI Web of Knowledge ${ }^{\text {sM }}$}

Journal Citation Reports ${ }^{\circledast}$

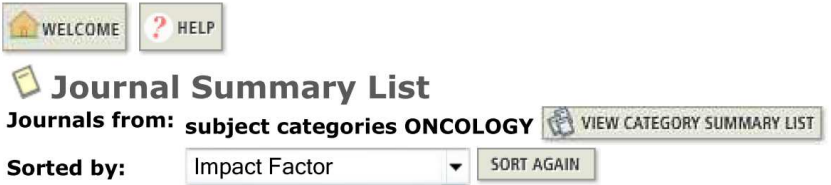

Page 1 of 10 MARK ALL UPDATE MARKED LIST Ranking is based on your journal and sort selections.

\begin{tabular}{|c|c|c|c|c|c|c|c|c|c|c|c|}
\hline \multirow[b]{2}{*}{ Mark } & \multirow[b]{2}{*}{ Rank } & \multirow{2}{*}{$\begin{array}{c}\text { Abbreviated Journal } \\
\text { Title } \\
\text { (linked to journal } \\
\text { information) }\end{array}$} & \multirow[b]{2}{*}{ ISSN } & \multicolumn{6}{|c|}{ JCR Data i } & \multicolumn{2}{|c|}{ Eigenfactor ${ }^{\circledR}$ Metrics $i$} \\
\hline & & & & \begin{tabular}{|l} 
Total \\
Cites
\end{tabular} & $\begin{array}{l}\text { Impact } \\
\text { Factor }\end{array}$ & \begin{tabular}{|l|} 
5-Year \\
Impact \\
Factor
\end{tabular} & $\begin{array}{c}\text { Immediacy } \\
\text { Index }\end{array}$ & Articles & $\begin{array}{c}\text { Cited } \\
\text { Half- } \\
\text { life } \\
\end{array}$ & \begin{tabular}{|c|}
$\begin{array}{c}\text { Eigenfactor } \\
\text { Score }\end{array}$ \\
\end{tabular} & $\begin{array}{c}\text { Article } \\
\text { Influence }{ }^{\circledR} \\
\text { Score }\end{array}$ \\
\hline$\square$ & 1 & CA-CANCER J CLIN & $\begin{array}{l}0007- \\
9235\end{array}$ & 10976 & 101.780 & 67.410 & 21.263 & 19 & 3.8 & 0.04500 & 24.536 \\
\hline 回 & 2 & NAT REV CANCER & \begin{tabular}{|l|}
$1474-$ \\
$175 x$ \\
\end{tabular} & 28602 & 37.545 & 38.460 & 4.838 & 68 & 5.8 & 0.12608 & 17.949 \\
\hline$\square$ & 3 & CANCER CELL & \begin{tabular}{|l}
$1535-$ \\
6108 \\
\end{tabular} & 19726 & 26.566 & 28.174 & 5.045 & 111 & 5.0 & 0.11452 & 15.267 \\
\hline$\square$ & 4 & LANCET ONCOL & \begin{tabular}{|l|}
$1470-$ \\
2045 \\
\end{tabular} & 13237 & 22.589 & 18.730 & 4.848 & 105 & 3.7 & 0.06914 & 7.421 \\
\hline$\square$ & 5 & J CLIN ONCOL & \begin{tabular}{|l|}
$0732-$ \\
$183 X$ \\
\end{tabular} & 120262 & 18.372 & 16.762 & 4.217 & 729 & 5.2 & 0.39463 & 5.560 \\
\hline Q & 6 & I NATL CANCER I & \begin{tabular}{|l|}
$0027-$ \\
8874 \\
\end{tabular} & 35847 & 13.757 & 14.537 & 3.308 & 130 & 9.5 & 0.08069 & 6.188 \\
\hline 回 & 7 & NAT REV CLIN ONCOL & \begin{tabular}{|l|}
$1759-$ \\
4774
\end{tabular} & 1558 & 11.963 & 12.000 & 3.477 & 65 & 1.9 & 0.00999 & 4.995 \\
\hline 回 & 8 & CANCER METAST REV & \begin{tabular}{|l|}
$0167-$ \\
7659
\end{tabular} & 4501 & 10.573 & 10.339 & 0.840 & 50 & 5.3 & 0.01804 & 3.919 \\
\hline$\square$ & 9 & LEUKEMIA & \begin{tabular}{|l|}
$8887-$ \\
6924 \\
\end{tabular} & 17255 & 9.561 & 8.205 & 2.126 & 183 & 5.4 & 0.06764 & 3.329 \\
\hline 回 & 10 & BBA-REV CANCER & \begin{tabular}{|l|}
$0304-$ \\
$419 x$
\end{tabular} & 3046 & 9.380 & 9.939 & 1.756 & 41 & 4.7 & 0.01246 & 3.795 \\
\hline 回 & 11 & NAT CLIN PRACT ONCOL & \begin{tabular}{|l|}
$1743-$ \\
4254 \\
\end{tabular} & 1527 & 8.000 & 7.327 & & 0 & 4.6 & 0.00869 & 2.822 \\
\hline 回 & 12 & CANCER RES & \begin{tabular}{|l|}
$0008-$ \\
5472 \\
\end{tabular} & 138772 & 7.856 & 8.164 & 1.582 & 749 & 7.5 & 0.36273 & 3.100 \\
\hline 回 & 13 & STEM CELLS & \begin{tabular}{|l|}
$1066-$ \\
50999 \\
\end{tabular} & 17241 & 7.781 & 8.612 & 0.990 & 206 & 4.4 & 0.08118 & 2.935 \\
\hline$\square$ & 14 & CLIN CANCER RES & \begin{tabular}{|l|}
$1078-$ \\
0432 \\
\end{tabular} & 60853 & 7.742 & 7.508 & 1.486 & 767 & 5.4 & 0.20597 & 2.538 \\
\hline 回 & 15 & J MAMMARY GLAND BIOL & \begin{tabular}{|l|}
$1083-$ \\
3021 \\
\end{tabular} & 1972 & 6.741 & 5.819 & 0.281 & 32 & 7.2 & 0.00559 & 2.117 \\
\hline 回 & 16 & ADV CANCER RES & \begin{tabular}{|l|}
$0065-$ \\
$230 X$ \\
\end{tabular} & 1838 & 6.733 & 5.766 & 0.263 & 19 & 9.7 & 0.00483 & 2.373 \\
\hline 回 & 17 & SEMIN CANCER BIOL & \begin{tabular}{|l|}
$1044-$ \\
$579 X$ \\
\end{tabular} & 3861 & 6.475 & 7.584 & 1.111 & 45 & 6.1 & 0.01336 & 2.998 \\
\hline 回 & 18 & ANN ONCOL & \begin{tabular}{|l|}
$0923-$ \\
7534
\end{tabular} & 19905 & 6.425 & 5.469 & 1.812 & 372 & 4.9 & 0.06614 & 1.797 \\
\hline 回 & 19 & ONCOGENE & \begin{tabular}{|l|}
$0950-$ \\
9232 \\
\end{tabular} & 58176 & 6.373 & 6.889 & 1.777 & 444 & 7.2 & 0.17168 & 2.820 \\
\hline 回 & 20 & J PATHOL & $\left|\begin{array}{l}0022- \\
3417\end{array}\right|$ & 13421 & 6.318 & 6.143 & 2.153 & 176 & 7.5 & 0.03406 & 2.232 \\
\hline
\end{tabular}

MARK ALL UPDATE MARKED LIST

Journals 1 - 20 (of 196) 


\section{ISI Web of Knowledge ${ }^{\text {SM }}$}

Journal Citation Reports ${ }^{\oplus}$

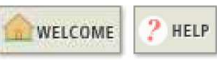

2011 JCR Science Edition

$\checkmark$ Journal Summary List

Journals from: subject categories ONCOLOGY RI VIEW CATEGORY SUMMARY LIST

$\underline{\text { Journal Title Changes }}$

Sorted by: Impact Factor $\quad$ SORT AGAIN

Journals 21 - 40 (of 196)

$\mid\langle\langle[\underline{1}|2| \underline{3}|\underline{4}| \underline{5}|\underline{6}| \underline{7}|\underline{8}| \underline{9} \mid \underline{10}]\rangle \Rightarrow\rangle$

Page 2 of 10 MARK ALL UPDATE MARKED LIST

Ranking is based on your journal and sort selections.

\begin{tabular}{|c|c|c|c|c|c|c|c|c|c|c|c|}
\hline \multirow[b]{2}{*}{ Mark } & \multirow[b]{2}{*}{ Rank } & \multirow{2}{*}{$\begin{array}{c}\text { Abbreviated Journal } \\
\text { Title } \\
\text { (linked to journal } \\
\text { information) }\end{array}$} & \multirow[b]{2}{*}{ ISSN } & \multicolumn{6}{|c|}{ JCR Data i } & \multicolumn{2}{|c|}{ Eigenfactor ${ }^{\circledR}$ Metrics $\mathrm{i}$} \\
\hline & & & & $\begin{array}{l}\text { Total } \\
\text { Cites }\end{array}$ & $\begin{array}{l}\text { Impact } \\
\text { Factor }\end{array}$ & \begin{tabular}{|c|} 
5-Year \\
Impact \\
Factor \\
\end{tabular} & $\underset{\text { Index }}{\text { Immediacy }}$ & Articles & \begin{tabular}{|l} 
Cited \\
Half- \\
life
\end{tabular} & $\begin{array}{c}\text { Eigenfactor } \\
\text { Score }\end{array}$ & $\begin{array}{c}\text { Article } \\
\text { Influence }{ }^{\circledR} \\
\text { Score } \\
\end{array}$ \\
\hline 回 & 21 & CANCER TREAT REV & $\begin{array}{l}0305- \\
7372\end{array}$ & 3642 & 6.054 & 5.828 & 1.000 & 67 & 4.2 & 0.01289 & 1.807 \\
\hline$\square$ & 22 & NEOPLASIA & $\begin{array}{l}1522- \\
8002\end{array}$ & 5282 & 5.946 & 5.133 & 1.712 & 111 & 4.6 & 0.01965 & 1.693 \\
\hline$\square$ & 23 & NEURO-ONCOLOGY & $\begin{array}{l}1522- \\
8517\end{array}$ & 3048 & 5.723 & 5.884 & 1.200 & 130 & 3.4 & 0.01253 & 1.935 \\
\hline$\square$ & 24 & CARCINOGENESIS & $\begin{array}{l}0143- \\
3334\end{array}$ & 20081 & 5.702 & 5.203 & 0.818 & 242 & 7.7 & 0.04645 & 1.700 \\
\hline 回 & 25 & RADIOTHER ONCOL & $\left|\begin{array}{l}0167- \\
8140\end{array}\right|$ & 10758 & 5.580 & 4.709 & 1.152 & 296 & 5.0 & 0.02558 & 1.155 \\
\hline$\square$ & 26 & EUR J CANCER & $\left|\begin{array}{l}0959- \\
8049\end{array}\right|$ & 19134 & 5.536 & 4.781 & 0.817 & 317 & 6.6 & 0.05306 & 1.646 \\
\hline 回 & 27 & INT J CANCER & $\left|\begin{array}{l}0020- \\
7136\end{array}\right|$ & 41471 & 5.444 & 4.919 & 1.444 & 603 & 6.7 & 0.11235 & 1.674 \\
\hline 回 & 28 & BREAST CANCER RES & $\begin{array}{l}1465- \\
542 x\end{array}$ & 6483 & 5.245 & 6.091 & 0.733 & 172 & 4.7 & 0.02648 & 2.185 \\
\hline 回 & 29 & MOL CANCER THER & $\begin{array}{l}1535- \\
7163\end{array}$ & 12886 & 5.226 & 5.390 & 0.885 & 226 & 4.3 & 0.05742 & 1.787 \\
\hline 回 & 30 & J ENVIRON SCI HEAL C & $\begin{array}{l}1059- \\
0501\end{array}$ & 396 & 5.160 & 5.623 & 0.250 & 12 & 4.0 & 0.00145 & 1.493 \\
\hline 回 & 31 & MOL ONCOL & $\begin{array}{l}1574- \\
7891\end{array}$ & 724 & 5.082 & & 1.542 & 48 & 2.6 & 0.00455 & \\
\hline 回 & 32 & PIGM CELL MELANOMA R & $\begin{array}{l}1755- \\
1471\end{array}$ & 2854 & 5.059 & 5.106 & 1.135 & 74 & 6.0 & 0.00890 & 1.839 \\
\hline 回 & 33 & BRIT J CANCER & $\begin{array}{l}0007- \\
0920\end{array}$ & 35335 & 5.042 & 4.955 & 0.749 & 541 & 7.5 & 0.08263 & 1.646 \\
\hline 回 & 34 & CANCER PREV RES & $\begin{array}{l}1940- \\
6207\end{array}$ & 2068 & 4.908 & 5.181 & 1.423 & 234 & 2.1 & 0.01037 & 1.708 \\
\hline 回 & 35 & ONCOTARGET & $\begin{array}{l}1949- \\
2553\end{array}$ & 493 & 4.784 & 4.784 & 1.099 & 121 & 1.3 & 0.00152 & 1.121 \\
\hline$\square$ & 36 & CANCER-AM CANCER SOC & $\left|\begin{array}{l}0008- \\
543 x\end{array}\right|$ & 59451 & 4.771 & 5.306 & 0.907 & 594 & $>10.0$ & 0.11317 & 1.926 \\
\hline$\nabla$ & 37 & BREAST CANCER RES TR & $\begin{array}{l}0167- \\
6806\end{array}$ & 12507 & 4.431 & 4.351 & 0.955 & 554 & 4.0 & 0.04431 & 1.392 \\
\hline 回 & 38 & CRIT REV ONCOL HEMAT & $\begin{array}{l}1040- \\
8428\end{array}$ & 4110 & 4.411 & 4.705 & 0.754 & 114 & 5.7 & 0.01184 & 1.464 \\
\hline$\square$ & 39 & J NATL COMPR CANC NE & $\begin{array}{l}1540- \\
1405\end{array}$ & 1414 & 4.409 & & 0.467 & 75 & 3.0 & 0.00952 & \\
\hline$\square$ & 40 & ENDOCR-RELAT CANCER & $\begin{array}{l}1351- \\
0088\end{array}$ & 3879 & 4.364 & 4.751 & 1.227 & 44 & 5.2 & 0.01503 & 1.659 \\
\hline
\end{tabular}

MARK ALL UPDATE MARKED LIST

Journals 21 - 40 (of 196) 


\section{Publikation}

Influence of CYP2D6 genotype on tamoxifen efficacy in advanced breast cancer

http://dx.doi.org/10.1007/s10549-013-2565-3 


\section{Lebenslauf}

"Mein Lebenslauf wird aus datenschutzrechtlichen Gründen in der elektronischen Version meiner Arbeit nicht veröffentlicht." 


\section{Publikationsliste}

Karle J, Bolbrinker J, Vogl S, et al. Influence of CYP2D6 genotype on tamoxifen efficacy in advanced breast cancer. Breast Cancer Res Treat 2013;139:553-60.

Impact Factor: 4,431

\section{Kongressbeitrag}

Regierer, A. C., Karle, J., Bolbrinker, J. et al., (2012). CYP2D6*4 genotype influences tamoxifen efficacy in advanced breast cancer. Freier Vortrag auf der Jahrestagung der Deutschen, Österreichischen und Schweizerischen Gesellschaften für Hämatologie und Onkologie, Stuttgart. Online im Internet: URL: https://m.dgho2012.de/abstract?aid=689. 


\section{Danksagung}

Ich möchte mich herzlich bedanken bei:

Herrn Prof. Dr. med. K. Possinger, dem Direktor der Medizinischen Klinik mit Schwerpunkt Hämatologie, Onkologie und Tumorimmunologie der Charité Campus Mitte, für die Bereitstellung des Themas und die Förderung dieser Arbeit,

Herrn Prof. R. Kreutz, dem Leiter des Instituts für Klinische Pharmakologie und Toxikologie der Charité Campus Mitte, für die Möglichkeit zur Durchführung der Arbeit in seiner Forschungsgruppe,

Frau Dr. med. Anne Constanze Regierer für die engagierte Betreuung, die zahlreichen Anregungen während der Entstehung dieser Arbeit sowie für ihre Motivation und Förderung,

Frau Dr. med. Juliane Bolbrinker und Frau Dr. rer. nat. Silvia Vogl für die praktische und theoretische Einarbeitung sowie die konstruktiven fachlichen Diskussionen bezüglich der Thematik dieser Dissertation,

meiner Schwester Janine Karle für ihre methodische Beteiligung und ihr großes Engagement,

Frau Karen Böhme und Frau Sabine Weickmann für die kompetenten Hilfestellungen bei Problemen und Fragestellungen im Labor,

Herrn Jörg Johannes, Frau Anja Derlet, Herrn Bastian Maroski und Frau Dr. med. Katja Grabowski für die humorvolle und nette Arbeitsatmosphäre,

Frau Dr. med. Annette Dieing, Frau Dr. med. J. Herrenberger, Frau Dr. med. Ch. Keitel-Wittig, Herr PD Dr. med. P. Kiewe, Frau Dr. med. I. Blau sowie den Mitarbeitern der MammaAmbulanz der Charité Campus Mitte für die Unterstützung bei der Patientenaufklärung und der Probengewinnung,

allen Patientinnen und Patienten, die diese Studie erst ermöglicht haben, 
Frau Katharina Kopetschke und Frau Katharina Schoofs für ihr offenes Ohr, die Aufmunterungen und Hilfe bei der abschließenden Fertigstellung der Arbeit,

Herrn Robert Pikulla für seine Geduld, Motivation sowie den unschätzbaren Rückhalt und nicht zuletzt meinen Eltern, die in jeglicher Hinsicht die Grundsteine für meinen Weg gelegt und mich mit aller Kraft unterstützt haben. 